\title{
Finite-Time Stability of Atangana-Baleanu Fractional-Order Linear Systems
}

\author{
Jiale Sheng, ${ }^{1}$ Wei Jiang $\mathbb{D}^{1},{ }^{1}$ and Denghao Pang $\mathbb{D}^{1,2}$ \\ ${ }^{1}$ School of Mathematical Sciences, Anhui University, Hefei 230601, China \\ ${ }^{2}$ School of Internet, Anhui University, Hefei 230601, China \\ Correspondence should be addressed to Wei Jiang; jiangwei@ahu.edu.cn and Denghao Pang; pangdh197@163.com
}

Received 16 July 2020; Revised 23 August 2020; Accepted 1 September 2020; Published 17 September 2020

Academic Editor: Atif Khan

Copyright (c) 2020 Jiale Sheng et al. This is an open access article distributed under the Creative Commons Attribution License, which permits unrestricted use, distribution, and reproduction in any medium, provided the original work is properly cited.

This paper investigates a fractional-order linear system in the frame of Atangana-Baleanu fractional derivative. First, we prove that some properties for the Caputo fractional derivative also hold in the sense of AB fractional derivative. Subsequently, several sufficient criteria to guarantee the finite-time stability and the finite-time boundedness for the system are derived. Finally, an example is presented to illustrate the validity of our main results.

\section{Introduction}

The topic of fractional calculus is more than three hundred years old. The number of applications of fractional calculus rapidly grows in recent decades. Fractional differential equations (FDEs) [1-7] have been successfully confirmed to be useful tools in various fields such as electrical circuits, diffusion, economy, and control problem.

Recently, many authors try to find new fractional operators with different kernels in order to better describe these phenomena. In 2015, Caputo and Fabrizi [8] present a new definition of fractional derivative with exponential kernel which is called CF fractional derivatives. In 2016, based on CF fractional derivatives, Atangana and Baleanu [9] introduced another new definition of fractional derivatives called $A B$ fractional derivatives with a nonlocal and nonsingular kernel which are built by the generalized Mittag-Leffler function. In addition, the study in [9] derived the fractional integral associate to $A B$ derivatives by taking the inverse Laplace transform and using the convolution theorem. The detail definitions of ABR fractional derivative, $\mathrm{ABC}$ fractional derivative, and $\mathrm{AB}$ fractional integral will be introduced in Section 2.

On the other hand, many basic properties of $A B$ fractional differintegral have already been studied in recent years [10-12], such as integration by parts, mean value theorem and Taylor's theorem, semigroup property, and product rule and chain rule. And some problems about existence of solution, stability, controllability, and optimal control are also studied by several researchers [13-18]. Having the advantage of nonlocal and nonsingular kernel, $\mathrm{AB}$ fractional derivatives have been widely applied in many fields such as diffusion equation [19], electromagnetic waves in dielectric media [20], chaos [21], and circuit model [22].

Finite-time stability (FTS) and finite-time boundedness (FTB) analysis is an important problem in control theory [23-29]. Since it always considers the behavior of systems in finite time, it is very useful in many practical application systems. Moreover, it should be noted that FTS and Lyapunov asymptotic stability are independent concepts. In [23], the authors have introduced the definition of FTS and FTB and given the sufficient condition for the FTB of linear timeinvariant (LTI) systems with integer order. The FTS and FTB for the fractional-order systems with $\alpha \in(0,1)$ have been given in [24]. Motivated by the above works, this paper studies the FTS and FTB for the class of fractional-order LTI systems in the sense of this new type of $A B$ fractional derivatives. First, we establish one new property of this new type of $A B$ fractional derivatives. Second, we introduce the concept of FTS and FTB for the fractional-order LTI system in the sense of $\mathrm{AB}$ fractional derivative corresponding with integer-order 
system and provide the sufficient conditions which guarantee FTS and FTB for a class of fractional-order LTI systems in the sense of $A B$ fractional derivative. Third, the problem of designing feedback controllers to keep FTS and FTB for fractional closed-loop systems in the sense of AB is studied.

Throughout this paper, $\mathfrak{R}^{n}$ denotes the $n$-dimensional Euclidean space, $\mathfrak{R}^{m \times n}$ is $m \times n$ real matrix, $A^{T}$ is the transpose of matrix $A, A^{-1}$ is the inverse of matrix $A$, $A>0(<0)$ indicates that the matrix $A$ is positive (negative) definite, and $\lambda_{\max }(A)$ and $\lambda_{\min }(A)$ are the maximum and minimum eigenvalues of matrix $A$.

\section{Preliminaries}

In this section, we present the definition of $\mathrm{AB}$ fractional derivatives and integral.

In addition, some properties of $\mathrm{AB}$ fractional calculus and Mittag-Leffler function are introduced.

Definition 1 (see [9]). Let $0<\alpha<1, f \in L^{1}(0, T)$, and $T>0$. Then, the $\mathrm{AB}$ fractional integral operator ${ }^{\mathrm{AB}} I_{t}^{\alpha}$ is defined by

$$
{ }^{\mathrm{AB}} I_{t}^{\alpha} f(t)=\frac{1-\alpha}{B(\alpha)} f(t)+\frac{\alpha}{B(\alpha)}\left(I_{t}^{\alpha} f\right)(t),
$$

where $\left(I_{t}^{\alpha} f\right)(t)=(1 / \Gamma(\alpha)) \int_{0}^{t}(t-s)^{(\alpha-1)} f(s) \mathrm{d} s$ is the classical Riemann-Liouville fractional integral, $B(\alpha)$ denotes a real-valued normalization function satisfying $B(\alpha)>0$, and $B(0)=B(1)=1$.

Definition 2 (see [9]). Let $0<\alpha<1, f \in L^{1}(0, T)$, and $T>0$. Then, the ABR fractional derivative of order $\alpha$ is defined by

$$
{ }^{\mathrm{ABR}} D_{t}^{\alpha} f(t)=\frac{B(\alpha)}{1-\alpha} \frac{\mathrm{d}}{\mathrm{d} t} \int_{0}^{t} E_{\alpha}\left(-\frac{\alpha}{1-\alpha}(t-s)^{\alpha}\right) f(s) \mathrm{d} s,
$$

where $E_{\alpha}$ is one-parameter Mittag-Leffler function denoted by $E_{\alpha}(t)=\sum_{n=0}^{\infty}\left(t^{n} / \Gamma(\alpha n+1)\right)$.

Definition 3 (see [9]). Let $0<\alpha<1, f \in L^{1}(0, T)$, and $T>0$. Then, the ABC fractional derivative of order $\alpha$ is defined by

$$
{ }^{\mathrm{ABC}} I_{t}^{\alpha} f(t)=\frac{B(\alpha)}{1-\alpha} \int_{0}^{t} E_{\alpha}\left(-\frac{\alpha}{1-\alpha}(t-s)^{\alpha}\right) f^{\prime}(s) \mathrm{d} s .
$$

In $[9,12]$, the authors have introduced some basic properties of $\mathrm{AB}$ fractional differintegral which will be used in this paper. For $0<\alpha<1$, the relation between $A B$ integral operators and $\mathrm{AB}$ differential operators is given as

$$
\begin{aligned}
{ }^{\mathrm{AB}} I_{t}^{\alpha}\left[{ }^{\mathrm{ABR}} D_{t}^{\alpha} f(t)\right] & =f(t), \\
{ }^{\mathrm{AB}} I_{t}^{\alpha}\left[{ }^{\mathrm{ABR}} D_{t}^{\alpha} f(t)\right] & =f(t)-f(0) .
\end{aligned}
$$

The Laplace transform of $\mathrm{AB}$ fractional derivative is defined as follows:

$$
L\left\{{ }^{\mathrm{ABC}} D_{t}^{\alpha} f(t) ; s\right\}=\frac{B(\alpha)}{1-\alpha} \cdot \frac{s^{\alpha} F(s)-s^{\alpha-1} f(0)}{s^{\alpha}+(\alpha / 1-\alpha)},
$$

where $F(s)=L\{f(t) ; s\}=\int_{0}^{\infty} e^{-s t} f(t) \mathrm{d} s$.
In addition, the Mittag-Leffler function which plays an important role in $\mathrm{AB}$ fractional derivative will appear frequently in this paper. It is indispensable to introduce some more properties of the Mittag-Leffler function. The generalized Mittag-Leffler functions (two parameters) are denoted by $E_{\alpha, \beta}(t)=\sum_{n=0}^{\infty}\left(t^{n} / \Gamma(\alpha n+\beta)\right), \quad \alpha, \beta>0$, and $t \in \mathfrak{R}$. For $\beta=1, E_{\alpha, 1}(t)=E_{\alpha}(t)$. The Laplace transform of the Mittag-Leffler function is given as

$$
L\left\{t^{\beta-1} E_{\alpha, \beta}\left(-\lambda t^{\alpha}\right) ; s\right\}=\frac{s^{\alpha-\beta}}{s^{\alpha}+\lambda} .
$$

In [30], Wang et al. provide some properties which are useful to prove Lemma 2. It is easy to note that $E_{\alpha}^{\prime}(t)=(1 / \alpha) E_{\alpha, \alpha}(t)$. So, we have

$$
\lambda(t-s)^{\alpha-1} E_{\alpha, \alpha}\left(-\lambda(t-s)^{\alpha}\right)=\frac{\mathrm{d}}{\mathrm{d} t} E_{\alpha}\left(-\lambda(t-s)^{\alpha}\right) .
$$

And for $0<\alpha<1$, the functions $E_{\alpha}(\cdot)$ and $E_{\alpha, \alpha}(\cdot)$ are nonnegative, and for $t=0$, we have $E_{\alpha}(0)=1$.

\section{Lemmas}

This section will introduce two important lemmas. In [29], Ye et al. have obtained the generalized Gronwall inequality in the sense of Caputo derivative which has wide applications in fractional differential equations. On this basis, Jarad et al. [15] proposed the Gronwall inequality in the frame of $\mathrm{AB}$ fractional derivative.

Lemma 1. Suppose that $\alpha>0, g(t)(1-(1-\alpha / B(\alpha)) h(t))^{-1}$ is a nonnegative, nondecreasing, and locally integrable function on $[a, b),(\alpha h(t) / B(\alpha))(1-(1-\alpha / B(\alpha)) h(t))^{-1}$ is nonnegative and bounded on $[a, b)$, and $x(t)$ is nonnegative and locally integrable on $[a, b)$ with

$$
x(t) \leq g(t)+h(t)\left({ }^{\mathrm{AB}} I_{t}^{\alpha} x\right)(t) .
$$

Then,

$$
x(t) \leq \frac{g(t) B(\alpha)}{B(\alpha)-(1-\alpha) h(t)} E_{\alpha}\left(\frac{\alpha h(t)(t-a)^{\alpha}}{B(\alpha)-(1-\alpha) h(t)}\right) .
$$

In [31], Norelys et al. present a new property for the Caputo fractional derivative that is $(1 / 2)_{t}^{C} D_{t}^{\alpha} x^{2}(t) \leq$ $x(t)_{t_{0}}^{C} D_{t}^{\alpha} x(t), t \geq t_{0}$ holds for a continuous and derivable function $x(t) \in \mathfrak{R}$, and $0<\alpha<1$. According to this, we can prove that this property also holds in the sense of $A B$ fractional derivative.

Lemma 2. Let $0<\alpha<1$ and $x(t) \in C(J ; \mathfrak{R})$ be a continuous and differentiable function. Then, for any $t \in J$, the following inequality holds:

$$
\frac{1}{2}{ }^{\mathrm{ABC}} D_{t}^{\alpha} x^{2}(t) \leq x(t)^{\mathrm{ABC}} D_{t}^{\alpha} x(t)
$$

Proof. By the definition of $\mathrm{AB}$ fractional derivative, we have 


$$
\begin{aligned}
x(t)^{\mathrm{ABC}} D_{t}^{\alpha} x(t) & =\frac{B(\alpha)}{1-\alpha} \int_{0}^{t} E_{\alpha}\left(-\frac{\alpha(t-s)^{\alpha}}{1-\alpha}\right) x(t) x^{\prime}(s) \mathrm{d} s, \\
\frac{1}{2}{ }^{\mathrm{ABC}} D_{t}^{\alpha} x^{2}(t) & =\frac{B(\alpha)}{1-\alpha} \int_{0}^{t} E_{\alpha}\left(-\frac{\alpha(t-s)^{\alpha}}{1-\alpha}\right) x(s) x^{\prime}(s) \mathrm{d} s .
\end{aligned}
$$

Equation (10) is equivalent to

$$
\frac{B(\alpha)}{1-\alpha} \int_{0}^{t} E_{\alpha}\left(-\frac{\alpha(t-s)^{\alpha}}{1-\alpha}\right)[x(t)-x(s)] x^{\prime}(s) \mathrm{d} s \geq 0 .
$$

Define $y(s)=x(t)-x(s)$. Then, equation (12) can be reformulated as

$$
-\frac{B(\alpha)}{1-\alpha} \int_{0}^{t} E_{\alpha}\left(-\frac{\alpha(t-s)^{\alpha}}{1-\alpha}\right) y(s) y^{\prime}(s) \mathrm{d} s \geq 0 .
$$

Let $u=(1 / 2) y^{2}$ and $v=(B(\alpha) / 1-\alpha) E_{\alpha}((-\alpha(t-$ $\left.\left.s)^{\alpha}\right) / 1-\alpha\right)$. By the integration by parts, equation (13) is equivalent to

$$
\begin{aligned}
& -\left.\frac{B(\alpha) y^{2}(s)}{2(1-\alpha)} E_{\alpha}\left(-\frac{\alpha(t-s)^{\alpha}}{1-\alpha}\right)\right|_{s=t}+\frac{B(\alpha) y^{2}(0)}{2(1-\alpha)} E_{\alpha}\left(-\frac{\alpha t^{\alpha}}{1-\alpha}\right) \\
& +\frac{B(\alpha)}{2(1-\alpha)} \int_{0}^{t} y^{2}(s) \alpha(t-s)^{\alpha-1} E_{\alpha, \alpha}\left(-\frac{\alpha(t-s)^{\alpha}}{1-\alpha}\right) \mathrm{d} s \geq 0 .
\end{aligned}
$$

Indeed, equation (14) holds obviously since $E_{\alpha}(0)=1$ and $y(t)=0$ deduce that the first term equals zero and the nonnegativity of $y^{2}(s), y^{2}(0), E_{\alpha}(\cdot)$, and $E_{\alpha, \alpha}(\cdot)$ leads to the nonnegativity of the latter two terms.

From Lemma 2, we can easily obtain the following two results.

Corollary 1. Let $0<\alpha<1$ and $x(t) \in C\left(J ; \mathfrak{R}^{n}\right)$ be a continuous and differentiable function on J. Then, for any $t \in J$, the following inequality holds:

$$
\frac{1}{2}{ }^{\mathrm{ABC}} D_{t}^{\alpha} x^{T}(t) x(t) \leq x^{T}(t)^{\mathrm{ABC}} D_{t}^{\alpha} x(t) .
$$

Proof. Assume that $x(t)=\left(x_{1}(t), x_{2}(t), \ldots, x_{n}(t)\right)^{T}$ and $x_{i}(t) \in C(J, \Re), i=1,2, \ldots, n$. By Lemma 2 , we have

$$
\begin{aligned}
\frac{1}{2}{ }^{\mathrm{ABC}} D_{t}^{\alpha} x^{T}(t) x(t) & =\frac{1}{2} \sum_{i=0}^{n}{ }^{\mathrm{ABC}} D_{t}^{\alpha} x_{i}(t) x_{i}(t) \\
& \leq \sum_{i=0}^{n} x_{i}(t)^{\mathrm{ABC}} D_{t}^{\alpha} x_{i}(t)=x^{T}(t)^{\mathrm{ABC}} D_{t}^{\alpha} x(t) .
\end{aligned}
$$

Corollary 2. Let $U \in \mathfrak{R}^{n \times n}$ be a symmetric positive definite matrix, $0<\alpha<1$, and $x(t) \in C\left(J ; \Re^{n}\right)$ be a continuous and differentiable function on $J$. Then, for any $t \in J$, the following inequality holds:

$$
\frac{1}{2}{ }^{\mathrm{ABC}} D_{t}^{\alpha} x^{T}(t) U x(t) \leq x^{T}(t) U^{\mathrm{ABC}} D_{t}^{\alpha} x(t) .
$$

Proof. From matrix theory, we know that there exists a nonsingular matrix $V$ such that $U=V^{T} V$. Define $y(t)=V x(t)$; then,

$$
\begin{aligned}
\frac{1}{2}{ }^{\mathrm{ABC}} D_{t}^{\alpha} x^{T}(t) U x(t) & =\frac{1}{2}{ }^{\mathrm{ABC}} D_{t}^{\alpha} y^{T}(t) y(t) \leq y^{T}(t)^{\mathrm{ABC}} D_{t}^{\alpha} y(t) \\
& =x^{T}(t) U^{\mathrm{ABC}} D_{t}^{\alpha} x(t) .
\end{aligned}
$$

\section{Main Results}

In this section, the concept of FTS and FTB for fractionalorder LTI systems in the sense of $\mathrm{AB}$ fractional derivative corresponding with integer-order systems is introduced. Several sufficient conditions of FTS and FTB for such systems are given.

Consider the following fractional order LTI system in the sense of $A B$ fractional derivative:

$$
\left\{\begin{array}{l}
{ }^{\mathrm{ABC}} D_{t}^{\alpha} x(t)=A x(t), \quad t \in J:=[0, T], \\
x(0)=x_{0}
\end{array}\right.
$$

where $0<\alpha<1, x(\cdot) \in \mathfrak{R}^{n}$, and $A \in \mathfrak{R}^{n \times n}$ is a constant matrix.

Definition 4. System (19) is said to be of finite-time stability with respect to $\left(r_{1}, r_{2}, R, T\right)$, with positive scalars $r_{1}, r_{2}, T$, $r_{2}>r_{1}$, and a matrix $R>0$, if

$$
x^{T}(0) R x(0) \leq r_{1} \Longrightarrow x^{T}(t) R x(t) \leq r_{2}, \quad t \in[0, T] .
$$

Theorem 1. Assume that there exists a scalar $\gamma>0$ and $a$ matrix $V \in \Re^{n \times n}, V>0$, satisfying

$$
U A+A^{T} U<\gamma U
$$

$$
\frac{\lambda_{\max }(V) B(\alpha)}{\lambda_{\min }(V)[B(\alpha)-\gamma(1-\alpha)]} E_{\alpha}\left(\frac{\alpha \gamma T^{\alpha}}{B(\alpha)-\gamma(1-\alpha)}\right)<\frac{r_{2}}{r_{1}}
$$

where $U=R^{(1 / 2)} V R^{(1 / 2)}$. Then, system (19) is FTS with respect to $\left(r_{1}, r_{2}, R, T\right)$.

Proof. Define the function $V(x(t))=x^{T}(t) U x(t)$. From Corollary 2 and condition equation (21), we have

$$
{ }^{\mathrm{ABC}} D_{t}^{\alpha} V(x(t)) \leq x^{T}(t)\left(U A+A^{T} U\right) x(t) \leq \gamma V(x(t)) .
$$

Then, there exists a nonnegative function $M(t)$ satisfying 


$$
\begin{gathered}
{ }^{\mathrm{ABC}} D_{t}^{\alpha} V(x(t))+\frac{1}{1-\alpha} \int_{0}^{t}(t-\tau)^{\alpha-1} E_{\alpha, \alpha} \\
\cdot\left(-\frac{\alpha(t-\tau)^{\alpha}}{1-\alpha}\right) M(\tau) \mathrm{d} \tau=\gamma V(x(t)) .
\end{gathered}
$$

Taking the Laplace transform on both sides of equality (24), we obtain

$$
\frac{B(\alpha)\left[s^{\alpha} V(x(s))-s^{\alpha-1} V(x(0))\right]}{(1-\alpha) s^{\alpha}+\alpha}+\frac{M(s)}{(1-\alpha) s^{\alpha}+\alpha}=\gamma V(x(t)) .
$$

It can be reformulated that

$$
\begin{aligned}
V(x(s))= & \frac{B(\alpha)}{B(\alpha)-\gamma(1-\alpha)} \cdot \frac{V(x(0)) s^{\alpha-1}}{s^{\alpha}-(\alpha \gamma /(B(\alpha)-\gamma(1-\alpha)))} \\
& -\frac{1}{B(\alpha)-\gamma(1-\alpha)} \cdot \frac{M(s)}{s^{\alpha}-(\alpha \gamma /(B(\alpha)-\gamma(1-\alpha)))}
\end{aligned}
$$

Applying the inverse Laplace transform, one has

$$
\begin{aligned}
V(x(t))= & \frac{B(\alpha) V(x(0))}{B(\alpha)-\gamma(1-\alpha)} E_{\alpha}\left(\frac{\alpha \gamma t^{\alpha}}{B(\alpha)-\gamma(1-\alpha)}\right) \\
& -\frac{1}{B(\alpha)-\gamma(1-\alpha)} \int_{0}^{t}(t-\tau)^{\alpha-1} E_{\alpha, \alpha} \\
& \cdot\left(\frac{\alpha \gamma(t-\tau)^{\alpha}}{B(\alpha)-\gamma(1-\alpha)}\right) M(\tau) \mathrm{d} \tau .
\end{aligned}
$$

Due to the nonnegativity of the second term in equation (27), it is easy to obtain

$$
V(x(t)) \leq \frac{B(\alpha) V(x(0))}{B(\alpha)-\gamma(1-\alpha)} E_{\alpha}\left(\frac{\alpha \gamma T^{\alpha}}{B(\alpha)-\gamma(1-\alpha)}\right) .
$$

Taking $V(x(t))=x^{T}(t) U x(t)$ and $U=R^{(1 / 2)} V R^{(1 / 2)}$ into equation (28) yields

$$
\begin{aligned}
x^{T}(t) R^{(1 / 2)} V R^{(1 / 2)} x(t) \leq & \frac{B(\alpha) x^{T}(0) R^{(1 / 2)} V R^{(1 / 2)} x(0)}{B(\alpha)-\gamma(1-\alpha)} E_{\alpha} \\
& \cdot\left(\frac{\alpha \gamma T^{\alpha}}{B(\alpha)-\gamma(1-\alpha)}\right) .
\end{aligned}
$$

This implies that

$$
\begin{aligned}
x^{T}(t) R x(t) \leq & \frac{\lambda_{\max }(V)}{\lambda_{\min }(V)} \cdot \frac{B(\alpha) x^{T}(0) R x(0)}{B(\alpha)-\gamma(1-\alpha)} E_{\alpha} \\
& \cdot\left(\frac{\alpha \gamma T^{\alpha}}{B(\alpha)-\gamma(1-\alpha)}\right) .
\end{aligned}
$$
that

According to condition equation (22), we can conclude

$$
x^{T}(0) R x(0) \leq r_{1} \Longrightarrow x^{T}(t) R x(t) \leq r_{2}
$$

For system (19), we also have the following results similar to Theorem 1 .

Corollary 3. Assume that there exists a scalar $\gamma>0$ and $a$ matrix $V \in \mathfrak{R}^{n \times n}, V>0$, satisfying

$$
U A^{T}+A U<\gamma U
$$

$$
\frac{\lambda_{\max }(V) B(\alpha)}{\lambda_{\min }(V)[B(\alpha)-\gamma(1-\alpha)]} E_{\alpha}\left(\frac{\alpha \gamma T^{\alpha}}{B(\alpha)-\gamma(1-\alpha)}\right)<\frac{r_{2}}{r_{1}},
$$

where $U=R^{(1 / 2)} V R^{(1 / 2)}$. Then, system (19) is FTS with respect to $\left(r_{1}, r_{2}, R, T\right)$.

Based on system (19), we will consider the finite-time stabilization of fractional-order systems in the AB fractional derivative sense with control function:

$$
\left\{\begin{array}{l}
{ }^{\mathrm{ABC}} D_{t}^{\alpha} x(t)=A x(t)+B u(t), \quad t \in J, \\
x(0)=x_{0}
\end{array}\right.
$$

where $u(t) \in \mathfrak{R}^{m}$ is a control function and $B \in \mathfrak{R}^{n \times m}$ is a constant matrix. We assume that all the state variables are available for state feedback. The problem is to design a state feedback controller $u(t)=K x(t)$, where $K \in \mathfrak{R}^{m \times n}$ is the control gain matrix to be designed, such that the closed-loop system is

$$
\left\{\begin{array}{l}
{ }^{\mathrm{ABC}} D_{t}^{\alpha} x(t)=A_{c} x(t), \quad t \in J, \\
x(0)=x_{0} .
\end{array}\right.
$$

where $A_{c}=A+B K$, being FTS with respect to $\left(r_{1}, r_{2}, R, T\right)$.

Theorem 2. Assume that there exists a scalar $\gamma>0$, a matrix $V \in \mathfrak{R}^{n \times n}, V>0$, and a matrix $L \in \mathfrak{R}^{m \times n}$ satisfying

$$
\begin{gathered}
A U+U A^{T}+B L+L^{T} B^{T}<\gamma U, \\
\frac{\lambda_{\max }(V) B(\alpha)}{\lambda_{\min }(V)[B(\alpha)-\gamma(1-\alpha)]} E_{\alpha}\left(\frac{\alpha \gamma T^{\alpha}}{B(\alpha)-\gamma(1-\alpha)}\right)<\frac{r_{2}}{r_{1}},
\end{gathered}
$$

where $U=R^{(1 / 2)} V R^{(1 / 2)}$. Then, under the feedback controller $u(t)=K x(t)=L U^{-1} x(t)$, system (33) is FTS with respect to $\left(r_{1}, r_{2}, R, T\right)$.

Proof. Apply the state feedback controller $u(t)=L U^{-1} x(t)$ to system (33) such that the corresponding closed-loop system is

$$
{ }^{\mathrm{ABC}} D_{t}^{\alpha} x(t)=\left(A+B L U^{-1}\right) x(t) .
$$

By Corollary 3, it is clear that system (34) is FTS under the condition equation (35) and equation (36).

Next, we will study the problem of FTB for fractionalorder LTI systems in the frame of AB fractional derivative. Consider the following systems: 


$$
\left\{\begin{array}{l}
{ }^{\mathrm{ABC}} D_{t}^{\alpha} x(t)=A x(t)+D w(t), \quad t \in J, \\
x(0)=x_{0},
\end{array}\right.
$$

where $w(t) \in \mathfrak{R}^{l}$ is the disturbance input and satisfies $w^{T}(t) w(t) \leq d, d \geq 0 . D \in \mathfrak{R}^{n \times l}$ is a constant matrix.

Definition 5. System (38) is said to be of finite-time boundedness with respect to $\left(r_{1}, r_{2}, R, T, d\right)$, with positive scalars $r_{1}, r_{2}, T, r_{2}>r_{1}$, and a matrix $R>0$, if

$$
\begin{array}{r}
x^{T}(0) R x(0) \leq r_{1} \Longrightarrow x^{T}(t) R x(t) \leq r_{2}, \\
t \in[0, T], \forall w: w^{T}(t) w(t) \leq d .
\end{array}
$$

Theorem 3. Assume that there exists a scalar $\gamma>0$ and two matrices $U_{1} \in \Re^{n \times n}, U_{1}>0$, and $V \in \mathfrak{R}^{l \times l}, V>0$, satisfying

$$
\begin{gathered}
\left(\begin{array}{cc}
A U+U A^{T}-\gamma U & D V \\
V D^{T} & -\gamma V
\end{array}\right)<0 \\
{\left[\frac{r_{1}}{\lambda_{\min }\left(U_{1}\right) K(\alpha)}+\frac{\gamma d M(\alpha)}{\lambda_{\min }(V) K(\alpha)}\right] E_{\alpha}\left(\frac{\alpha \gamma T^{\alpha}}{K(\alpha)}\right)<\frac{r_{2}}{\lambda_{\max }\left(U_{1}\right)},} \\
K(\alpha)=B(\alpha)-\gamma(1-\alpha)>0 .
\end{gathered}
$$

where $U=R^{-(1 / 2)} U_{1} R^{-(1 / 2)}$ and $M(\alpha)=(1-\alpha / B(\alpha))+$ $\left(T^{\alpha} /(B(\alpha) \Gamma(\alpha))\right)$. Then, system (38) is FTB with respect to $\left(r_{1}, r_{2}, R, T, d\right)$.

Proof. Let $V(x(t))=x^{T}(t) U^{-1} x(t)$, and taking $\mathrm{AB}$ fractional derivative of $V(x(t))$, by Corollary 2, we have

$$
\begin{aligned}
{ }^{\mathrm{ABC}} D_{t}^{\alpha} V(x(t)) & \leq x^{T}(t) U^{-1}(A x(t)+D w(t))+(A x(t)+D w(t))^{T} U^{-1} x(t) \\
& =\left(x^{T}(t) w^{T}(t)\right)\left(\begin{array}{cc}
U^{-1} A+A^{T} U^{-1} & U^{-1} D \\
D^{T} U^{-1} & 0
\end{array}\right)\left(\begin{array}{c}
x(t) \\
w(t)
\end{array}\right) .
\end{aligned}
$$

By premultiplying and postmultiplying equation (40) by the symmetric positive definite matrix $\left(\begin{array}{cc}U^{-1} & 0 \\ 0 & V^{-1}\end{array}\right)$, we have

$$
\left(\begin{array}{cc}
U^{-1} A+A U^{-1}-\gamma U^{-1} & U^{-1} D \\
D^{T} U^{-1} & -\gamma V^{-1}
\end{array}\right)<0 .
$$

Then, combining equations (43) and (44) leads to

$$
\begin{aligned}
{ }^{\mathrm{ABC}} D_{t}^{\alpha} V(x(t)) & \leq\left(x^{T}(t) w^{T}(t)\right)\left(\begin{array}{cc}
\gamma U^{-1} & 0 \\
0 & \gamma V^{-1}
\end{array}\right)\left(\begin{array}{c}
x(t) \\
w(t)
\end{array}\right) \\
& =\gamma V(x(t))+\gamma w^{T}(t) V^{-1} w(t) \\
& \leq \gamma V(x(t))+\frac{\gamma d}{\lambda_{\min }(V)} .
\end{aligned}
$$

Integrating with order $\alpha$ from 0 to $t$ in the frame of $\mathrm{AB}$ fractional integral on both sides of equality (45), then

$$
\begin{aligned}
V(x(t)) \leq & V(x(0))+{ }^{A B} I_{t}^{\alpha}\left(\frac{\gamma d}{\lambda_{\min }(V)}\right)+\gamma^{A B} I_{t}^{\alpha} V(x(t)) \\
= & V(x(0))+\frac{1-\alpha}{B(\alpha)} \cdot \frac{\gamma d}{\lambda_{\min }(V)}+\frac{\alpha \gamma d}{B(\alpha) \Gamma(\alpha) \lambda_{\min }(V)} \\
& \cdot \int_{0}^{t}(t-s)^{\alpha-1} \mathrm{~d} s+\gamma^{A B} I_{t}^{\alpha} V(x(t)) \\
\leq & V(x(0))+\frac{\gamma d M(\alpha)}{\lambda_{\min }(V)}+\gamma^{A B} I_{t}^{\alpha} V(x(t)),
\end{aligned}
$$

where $M(\alpha)=(1-\alpha / B(\alpha))+\left(T^{\alpha} /(B(\alpha) \Gamma(\alpha))\right)$. By equation (42) and applying Lemma 1 , we have

$$
\begin{aligned}
V(x(t)) \leq & {\left[\frac{V(x(0))}{B(\alpha)-\gamma(1-\alpha)}+\frac{\gamma d M(\alpha)}{\lambda_{\min }(V)(B(\alpha)-\gamma(1-\alpha))}\right] } \\
& \cdot E_{\alpha}\left(\frac{\alpha \gamma t^{\alpha}}{B(\alpha)-\gamma(1-\alpha)}\right)(1-\alpha) \\
= & {\left[\frac{V(x(0))}{K(\alpha)}+\frac{\gamma d M(\alpha)}{\lambda_{\min }(V) K(\alpha)}\right] E_{\alpha}\left(\frac{\alpha \gamma T^{\alpha}}{K(\alpha)}\right) . }
\end{aligned}
$$

It follows from $U=R^{-(1 / 2)} U_{1} R^{-(1 / 2)}$ that 


$$
\begin{aligned}
V(x(t)) & =x^{T}(t) U^{-1} x(t)=x^{T}(t) R^{-(1 / 2)} U_{1} R^{-(1 / 2)} x(t) \\
& \geq \frac{x^{T}(t) R x(t)}{\lambda_{\max }\left(U_{1}\right)}, \\
V(x(0)) & =x^{T}(0) U^{-1} x(0)=x^{T}(0) R^{-(1 / 2)} U_{1} R^{-(1 / 2)} x(0) \\
& \leq \frac{x^{T}(t) R x(t)}{\lambda_{\min }\left(U_{1}\right)} .
\end{aligned}
$$

Combining equations (47), (48), and (49), it follows that $\frac{x^{T}(t) R x(t)}{\lambda_{\max }\left(U_{1}\right)} \leq\left[\frac{r_{1}}{\lambda_{\min }\left(U_{1}\right) K(\alpha)}+\frac{\gamma d M(\alpha)}{\lambda_{\min }(V) K(\alpha)}\right] E_{\alpha}\left(\frac{\alpha \gamma T^{\alpha}}{K(\alpha)}\right)$.

By the condition equations (41) and (50), it implies that

$$
x^{T}(t) R x(t) \leq r_{2}, \quad t \in[0, T] .
$$

Now, we consider the problem of designing state feedback controllers $u(t)=K x(t)$ to stabilize the FTB problem for fractional-order LTI systems in the frame of AB fractional derivative:

$$
\left\{\begin{array}{l}
{ }^{\mathrm{ABC}} D_{t}^{\alpha} x(t)=A x(t)+B u(t)+D w(t), \quad t \in J \\
x(0)=x_{0}
\end{array}\right.
$$

Theorem 4. Assume that there exist a scalar $\gamma>0$, two matrices $U_{1} \in \mathfrak{R}^{n \times n}, U_{1}>0$, and $V \in \mathfrak{R}^{l \times l}, V>0$, and $a$ matrix $L \in \Re^{m \times n}$ satisfying equations (41) and (42) and

$$
\left(\begin{array}{cc}
A U+U A^{T}+B L+L^{T} B^{T}-\gamma U & D V \\
V D^{T} & -\gamma V
\end{array}\right)<0
$$

where $U=R^{-(1 / 2)} U_{1} R^{-(1 / 2)}$. Then, under the feedback controller $u(t)=K x(t)=L U^{-1} x(t)$, system (53) is FTS with respect to $\left(r_{1}, r_{2}, R, T, d\right)$.

Proof. Apply the state feedback controller $u(t)=L U^{-1} x(t)$ to system (53) such that the corresponding closed-loop system is

$$
{ }^{\mathrm{ABC}} D_{t}^{\alpha} x(t)=\left(A+B L U^{-1}\right) x(t)+D w(t) .
$$

By Theorem 3, it is clear that system (52) is FTB under the condition equations (41), (42), and (53).

\section{Example}

An example is presented to illustrate the main results.

Example 1. Consider the following fractional-order LTI system in the sense of $\mathrm{AB}$ fractional derivative with a disturbance defined by

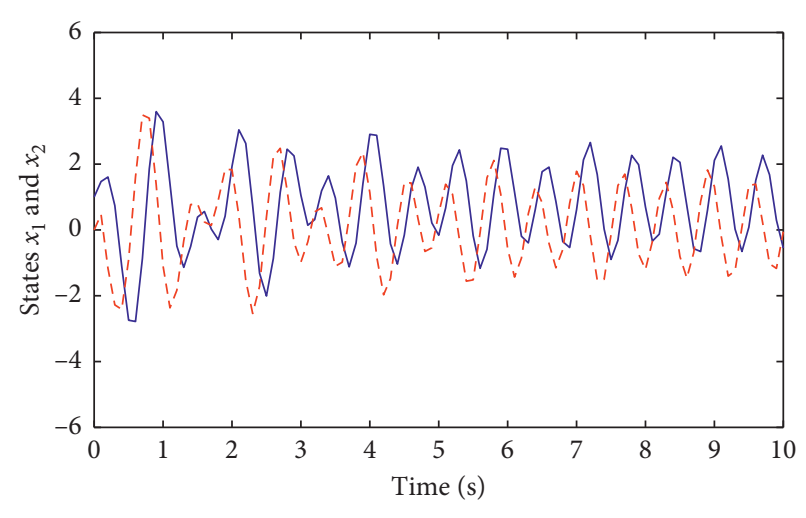

$\begin{aligned}- & x_{1} \\ --- & x_{2}\end{aligned}$

Figure 1: The state $x(t)$ of system (55) versus time.

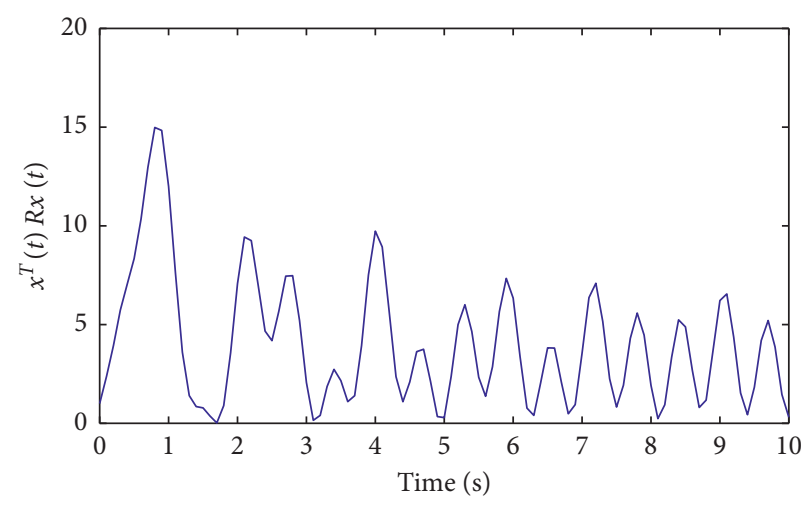

Figure 2: The trajectory of $x^{T}(t) R x(t)$ of system (55) versus time.

$$
{ }^{\mathrm{ABC}} D_{t}^{(1 / 2)} x(t)=\left(\begin{array}{cc}
1 & 1 \\
-1 & 1
\end{array}\right) x(t)+\left(\begin{array}{ll}
1 & 0 \\
0 & 1
\end{array}\right) w(t)
$$

where the initial value $x(0)=\left(\begin{array}{ll}1 & 0\end{array}\right)^{T}$ and the disturbance $w(t)=((\sin (t)-1) \cos (t))^{T}$. The parameters are given as $c_{1}=1, c_{2}=20, d=2, T=10, R=I$, and $B(\alpha)=1$. Applying the condition equations (40), (41), and (42) of Theorem 3 and setting $\gamma=0.05$, we can obtain the feasible matrices $U_{1}$ and $V$ as follows:

$$
\begin{aligned}
U_{1} & =\left(\begin{array}{cc}
0.97 & 0 \\
0 & 0.97
\end{array}\right), \\
V & =\left(\begin{array}{cc}
0.55 & 0 \\
0 & 0.55
\end{array}\right) .
\end{aligned}
$$

Therefore, system (55) is FTB with respect to $(1,20, I, 10,2)$. The state trajectory over $0-10 s$ with the initial state $x(0)=\left(\begin{array}{ll}1 & 0\end{array}\right)^{T}$ is shown in Figure 1. It is easy to see that system (55) is FTB with respect to $(1,20, I, 10,2)$ from Figure 2.

\section{Conclusion}

The contribution of this paper is to present one new property of $A B$ fractional derivatives and to provide the sufficient 
conditions which guarantee FTS and FTB for a class of fractional-order LTI systems in the sense of $\mathrm{AB}$ fractional derivative, as well as to study the problem of designing feedback controllers. Because of the interesting nonsingular kernel of this new fractional derivative, there is much work on this type of fractional calculus that is worth thinking about and discussing in the future.

\section{Data Availability}

No data were used to support this study.

\section{Conflicts of Interest}

The authors declare that they have no conflicts of interest.

\section{Authors' Contributions}

The authors contributed equally to the manuscript. All authors have read and approved the final manuscript..

\section{Acknowledgments}

This work was supported by the Natural Science Foundation of Anhui Province (2008085QA19) and National Natural Science Foundation of China (110131013 and 11471015).

\section{References}

[1] A. A. Kilbas, H. M. Srivastava, and J. J. Trujillo, Theory and Applications of Fractional Differential Equations, Elsevier, Amsterdam, The Netherland, 2006.

[2] K. Diethelm, The Analysis of Fractional Differential Equations, Springer, Berlin, Germany, 2010.

[3] Y. Zhou, Fractional Evolution Equations and Inclusions: Analysis and Control, Academic Press, Cambridge, MA, USA, 2016.

[4] I. Podlubny, Fractional Differential Equations, Academic Press, Cambridge, MA, USA, 1999.

[5] D. H. Pang, W. Jiang, S. Liu, and U. K. N. Azmat, "Wellposedness and iterative formula for fractional oscillator," Mathematical Methods in the Applied Sciences, pp. 1-13, 2019.

[6] H. Zhang, M. Ye, J. Cao, and A. Alsaedi, "Synchronization Control of Riemann-Liouville Fractional Competitive Network Systems with Time-varying Delay and Different Time Scales," International Journal of Control, Automation and Systems, vol. 16, no. 3, pp. 1404-1414, 2018.

[7] H. Zhang, R. Ye, J. Cao, A. Alsaedi, A. Alsaedie, and X. D. Li, "Existence and globally asymptotic stability of equilibrium solution for fractional-order hybrid BAM neural networks with distributed delays and impulses," Complexity, vol. 2017, Article ID 6875874, 9 pages, 2017.

[8] M. Caputo and M. Fabrizio, "A new definition of fractional derivative withoutSingular kernel," Progress in Fractional Differentiation and Applications, vol. 2, pp. 73-85, 2015.

[9] A. Atangana and D. Baleanu, "New fractional derivatives with nonlocal and non-singular kernel: theory and application to heat transfer model," Thermal Science, vol. 20, no. 2, pp. 763-769, 2016.

[10] T. Abdeljawad and D. Baleanu, "Integration by parts and its applications of a new nonlocal fractional derivative with Mittag-Leffler nonsingular kernel," The Journal of Nonlinear Sciences and Applications, vol. 10, no. 3, pp. 1098-1107, 2017.
[11] A. Fernandez and D. Baleanu, "The mean value theorem and Taylor's theorem for fractional derivatives with Mittag-Leffler kernel," Advances in Difference Equations, vol. 2018, no. 1, Article ID 86, 2018.

[12] D. Baleanu and A. Fernandez, "On some new properties of fractional derivatives with Mittag-Leffler kernel," Communications in Nonlinear Science and Numerical Simulation, vol. 59, pp. 444-462, 2018.

[13] J. Hristov, "On the atanganaaleanu derivative and its relation to the fading memory concept: the diffusion equation formulation" in Fractional Derivatives with Mittag-effler Kernel. Studies in Systems, Decision and Control, J. G'omez, L. Torres, and R. Escobar, Eds., Springer, Cham, Switzerland, 2019.

[14] J. Hristov, "Response functions in linear viscoelastic constitutive equations and related fractional operators," Mathematical Modelling of Natural Phenomena, vol. 14, no. 3, pp. 305-34, 2019.

[15] F. Jarad, T. Abdeljawad, and Z. Hammouch, "On a class of ordinary differential equations in the frame of AtanganaBaleanu fractional derivative," Chaos, Solitons \& Fractals, vol. 117, pp. 16-20, 2018.

[16] D. Aimene and D. Baleanu, "Controllability of semilinear impulsive Atangana-Baleanu fractional differential equations with delay," Chaos, Solitons \& Fractals, vol. 128, pp. 51-57, 2019.

[17] C. Seba, K. Logeswari, and F. Jarad, "New results on existence in the framework of Atangana-Baleanu derivative for fractional integro-differential equations," Chaos, Solitons \& Fractals, vol. 125, pp. 194-200, 2019.

[18] G. M. Bahaa, "Optimal control problem for variable-order fractional differential systems with time delay involving Atangana-Baleanu derivatives," Chaos, Solitons \& Fractals, vol. 122, pp. 129-142, 2019.

[19] J. F. Gómez-Aguilar, "Space-time fractional diffusion equation using a derivative with nonsingular and regular kernel," Physica A: Statistical Mechanics and its Applications, vol. 465, pp. 562-572, 2017.

[20] J. F. Gómez-Aguilar, R. F. Escobar-Jiménez, M. G. LópezLópez, and V. M. Alvarado-Martínez, "Atangana-Baleanu fractional derivative applied to electromagnetic waves in dielectric media," Journal of Electromagnetic Waves and Applications, vol. 30, no. 15, p. 1937, 2016.

[21] A. Atangana and I. Koca, "Chaos in a simple nonlinear system with Atangana-Baleanu derivatives with fractional order," Chaos, Solitons \& Fractals, vol. 89, pp. 447-454, 2016.

[22] B. S. T. Alkahtani, "Chua's circuit model with AtanganaBaleanu derivative with fractional order," Chaos, Solitons \& Fractals, vol. 89, pp. 547-551, 2016.

[23] F. Amato, M. Ariola, and P. Dorato, "Finite-time control of linear systems subject to parametric uncertainties and disturbances," Automatica, vol. 37, no. 9, pp. 1459-1463, 2001.

[24] Y.-j. Ma, B.-w. Wu, and Y.-E. Wang, "Finite-time stability and finite-time boundedness of fractional order linear systems," Neurocomputing, vol. 173, pp. 2076-2082, 2016.

[25] M. Li and J. Wang, "Exploring delayed Mittag-Leffler type matrix functions to study finite time stability of fractional delay differential equations," Applied Mathematics and Computation, vol. 324, pp. 254-265, 2018.

[26] R. Wu, Y. Lu, and L. Chen, "Finite-time stability of fractional delayed neural networks," Neurocomputing, vol. 149, pp. 700-707, 2015.

[27] M. P. Lazarevi and A. M. Spasi, "Finite-time stability analysis of fractional order time-delay systems: gronwall's approach," 
Mathematical and Computer Modelling, vol. 49, no. 3-4, pp. 475-481, 2009.

[28] D. Pang and W. Jiang, "Finite-time stability analysis of fractional singular time-delay systems," Advances in Difference Equations, vol. 2014, no. 1, Article ID 259, 2014.

[29] H. Ye, J. Gao, and Y. Ding, "A generalized Gronwall inequality and its application to a fractional differential equation," Journal of Mathematical Analysis and Applications, vol. 328, no. 2, pp. 1075-1081, 2007.

[30] J. Wang, M. Fečkan, and Y. Zhou, "Presentation of solutions of impulsive fractional Langevin equations and existence results," The European Physical Journal Special Topics, vol. 222, no. 8, pp. 1857-1874, 2013.

[31] A. C. Norelys, A. Manuel, and A. Javier, "Lyapunov functions for fractional order systems," Communications in Nonlinear Science and Numerical Simulation, vol. 19, pp. 2951-2957, 2014. 